\title{
The Influential Factors of Antisocial Personality Disorder
}

\begin{abstract}
Xuanxin Wen
Vanke Meisha Academy, Shenzhen, Guangdong, 518000, China

Corresponding author.Email: wenxuanxin@stu.vma.edu.cn

ABSTRACT

Antisocial Personality Disorder (ASPD) is a personality disorder characterized by a disregard for rules and laws, social norms, and lack of sympathy and remorse for others. It is caused by different reasons. Patients often resist treatment because they lose their self-awareness. Patients with severe symptoms even refuse to admit that they have a serious problem, and drugs may be misused. The treatment of ASPD is not well developed at present. Understanding the causes of diseases contributes to the formation and development of different treatments. This paper reviews and analyses the relationship between environmental and genetic factors and the development of antisocial personality disorder. The purpose of this review is to identify potential factors that may lead to anti-social personality disorder. The result shows that both environmental and genetic factors contribute to the development of ASPD, especially family factors and both have about a $50 \%$ chance of causing the disorder.
\end{abstract}

Keywords: Antisocial Personality Disorder, Personality, Psychology, Environment, Gene

\section{INTRODUCTION}

The Diagnostic and Statistical Manual of Mental Disorders (DSM 5) classifies all personality disorders into three main clusters. The antisocial disorder is located in cluster B which is called the dramatic, emotional, and erratic cluster including other personality disorders such as borderline personality disorder [1]. The diagnosis for antisocial personality disorder is based on psychological evaluation including thoughts, feelings, relationships, behavior patterns and family history, personal and medical history and relevant symptoms. These symptoms include behaviors containing performing acts that are grounds for arrest, repeated lying, fights, or assaults, disregard for the safety of oneself and others, failure to sustain consistent work behavior, and mistreating other individuals [2]. Some of the symptoms may occur during children's young ages. These signs during childhood can be evidence that helps the diagnosis [3].

Besides, antisocial personality disorder also has a high rate for combining other psychiatric diseases such as depression, bipolar and borderline personality disorder [2]. Many researchers have classified the various causes of antisocial personality disorder. It is difficult to completely separate environmental and genetic influences. It has been reported that the stability of antisocial behavior is due to genetic and common environmental influences [4]. However, there is still a lot of research on these two possible causes of the disease. A clearer understanding of the relationship between antisocial personality disorder and environment and heredity will help the medical community to better diagnose and treat it. Based on the previous studies on the causes of ASPD, the paper analyses genetic and environmental factors. The review of the main causes of the disease may provide new insights into the development of treatment for the disease. It will provide a basis for more detailed in-depth future researches.

\section{ANALYSIS ON THE CAUSES OF ANTISOCIAL PERSONALITY DISORDER}

Antisocial personality disorder has been paid close attention to by the research community for a long time. For a long time, many scholars have discussed and classified the causes of it from their professional perspective. There are many reasons for it, which can be divided into biological level and natural level. Many researchers have already classified different kinds of causes of the formation of antisocial personality disorder. Genes, brain differences and environmental factors can all be attributed to the formation and development of antisocial personality disorder. Despite different special brain structures, genetic and environmental factors are 
sometimes controversial since genes may contribute to half of the variance, and the environment will contribute to the other half in antisocial behavior.

In many antisocial personality disorder cases, it is hard to completely tease apart the influence of environment and genes [2]. There are reports that the stability of antisocial behavior is due to genetics and shared environmental influences [4]. However, there are still many studies about these two factors that can contribute to the disorder. According to previous studies, although both biological and psychological factors cannot fully explain the diseases' causes they can still be significant elements to cause and help develop ASPD. There are now more recent studies that have deeply. Among these factors, the author focuses on the specific relationship between genetic and environmental factors and antisocial personality disorder.

\subsection{Genetic factors}

\subsection{1 brief introduction of Genetic factors}

Gene is a biological concept. Gene factor is to explore the relationship between them from the perspective of biology. Genetic factors are the causes or conditions of the phenomenon that enable offspring to obtain genetic information from their parents. The expression of genetic factors mainly includes genes, specific genes and chromosome aberration. It has been proved that genetic factors account for about half of the differences in antisocial behavior [2]. Specific genes can attribute to the form and development of antisocial behaviors and antisocial personalities.

\subsection{2 correlational study}

A study with 240 children who suffered from attention deficit hyperactivity disorder found that children with a valine/methionine variant in the Catechol-O-Methyltransferase (COMT) gene showed stronger antisocial behavior than children without the variant. In addition to the COMT gene, the MAOA gene located on the X-chromosome can also be a specific gene that can attribute to the development of antisocial personality. In 2002, there was a longitudinal study made by Caspi et al. Proved result that men with low MAOA activity genotype were significantly more likely to exhibit child behavior disorders and adult antisocial behavior, including criminal arrest as adults. Although a low MAOA genotype does not increase the development of ASPD, its presence appears to put individuals at risk for ASPD, which can be triggered by abuse in the family [5]. Besides, there was also a study made by Basoglu et al. found that two variants of the symptomaticallyassociated protein 25 (SNAP25) gene were more frequently present in male participants with ASPD [2]. Although genes do not directly causes the diseases, there is still genetic evidence that can help explain the development of the disorder.

The specific genotype and variants for different specific genes are considered as genetic causes and evidence for ASPD, which will contribute to the maintenance and development of the disorder. Adoption studies can also be the evidence to prove the influence of genetic factors. Results from an adoption study showed that psychiatric records of biological relatives of adopters with mental illness were tested and the incidence of psychiatric illness was significantly higher than that of biological and adoptive relatives or control adopters. And biological relatives of criminal adopters were found to have higher crime rates than biological and adopted relatives of adopters or control adopters. It can be proved that criminal behavior by biological parents is a risk factor for children to commit crimes [6]-[7]. Besides, according to the study made by Berg-Nielsen and Wichström, parents' antisocial behavior poses risks to older children and adolescents. They found that parents' symptoms of personality disorders (antisocial, borderline, or narcissistic) explained $13.2 \%$ of the differences in children's behavioral symptoms [2].

\subsection{Environment factors}

\subsection{1 brief introduction of environment factors}

The environment is also a significant factor that can lead to the development of ASPD. In addition, it is always combined with genetic factors. For environmental factors, the family has always been a key element. For example, adverse childhood experiences, including various forms of abuse, neglect, and poverty in childhood can be considered as an environmental factor that helps the development of the disorder. According to studies held by Fergusson et al., the prevalence of ASPD was two to four times higher among 18 to 21 -year-olds and 21 to 25 -year-olds than among those who had not been sexually abused [25]. Similarly, those who experienced regular or severe physical abuse were two to seven times more likely to develop antisocial personality disorder than those who were not physically abused. Those who had witnessed or experienced violent crime, sexual and physical abuse, and other adverse childhood experiences were increased by 1.47 rate of having the disease to develop antisocial personality disorder [8]

\subsubsection{Family environment and negative childhood experiences}

Children of parents with antisocial personalities are more likely to experience abuse in childhood or witness domestic violence. Data from an epidemiological survey suggests that experiencing or witnessing intimate partner violence during childhood increases the risk of intimate partner violence in adulthood [2]. Moreover, according 
to Widom, abused or neglected children are more likely to be arrested as teenagers and adults. It can be proved that family environment and negative childhood experiences can influence the development of the disorder to some extent. In addition, parenting attachments during the early ages can also be a environmental factor. According to Shi et al., it is found that the parent-infant relationship during the first 18 months of life was a severe and significant predictor of ASPD in adulthood. Some maternal behaviors, such as quiet interactions, not greeting the baby, and comforting the baby with toys rather than herself, have also been found to predict later antisocial personality disorder outcomes. At age 8, disorganized attachment predicted later antisocial personality disorder [2]. It is proved that parents have played significant roles in causing and leading to children's ASPD in their future lives.

Besides, the twin studies have shown that criminality shows MZ-DZ differences in concordance. Given that identical, (identical, MZ) twins share the same genetic material, whereas fraternal (fraternal, DZ) twins share about half their genetic material, but (presumably) similar environment, it is concluded that the $M Z$ twins late in should behave twice as well as the DZ twins [5][9]. Although the twins shared the same genetic material, different growing environments can also cause differences in their rate of developing the disorder when they grow up. Shared environments can also be a cause for the development of the disease. In 1996, Thapar and McGuinn, from the sample of 198 pairs of same-sex twins, concluded that the spread of antisocial symptoms were entirely explained by shared environmental factors [10]. However, twin studies also showed that individuals that have different genetic backgrounds may have different responses to environmental influences [11].

\section{DISCUSSION}

Current research from different studies and researchers have shown that ASPD will be caused by many different kinds of reasons. For genetic factors, some specific genes' variants in different parts can cause the variances of the illness for different patients. However, genes will not be the direct cause of the disorder, and genetic causes about specific genes are not completely researched by researchers currently. Consequently, the area of the gene is also developed. Biological parents and relatives can also be considered a genetic factor. Relatives' diagnosis of ASPD can bring risks to their future children. The genetic materials that are inherited can also cause the variances of different children's illnesses.

For environmental factors, childhood experiences can be a very significant cause. Neglected, sexual abuse and physical abuse can be some kinds of negative childhood experiences. Witnessing violent crimes can also bring risks to children to suffer from ASPD. In early childhood, infant-mother relationships can influence the personality development of children when they grow older. Different growing environments and shared environments can be proved to be other significant environmental factors. The exact etiology of ASPD is still being developed by researchers. Neither environment nor genetic factors can fully account for the development and formation of the disorder. However, they can all be significant elements for the researchers to better develop appropriate therapies for the disorder and later on help more patients.

\section{CONCLUSION}

The result of this analysis are as follows: environmental factors and genetic factors are two factors related to antisocial personality disorder. Among the environmental factors, family factors play a major role. Specifically, if a child grows up in an disharmonious family, or fails to get the care of his parents for a long time at the age when he needs attachment or care most, what's more, he is likely to have the tendency of antisocial personality disorder when he is neglected for a long time according the analyses above. In the analysis of gene factors, specific genotype and variants of specific genes are very important factors. Gene factor explains its association with antisocial personality disorder from the perspective of physiology. The limitation of this study is that most of the references about ASPD and environmental factors contain references about twin studies, but the studies were done during a relatively early period. Some of the results may change over time. Using only previous studies might compromise the accuracy of the results. Future research will focus more on the scope of the experiment and the more accurate data.

\section{ACKNOWLEDGMENT}

First of all, I would like to express my deep gratitude to the teacher who gave me great help in the process of writing this paper. They gave me very pertinent suggestions and guidance in the structure of the paper and the establishment of the overall outline, so that my paper can proceed smoothly. Secondly, I want to thank my parents and classmates. They gave me great suggestions and affirmation during the conception of my paper, and also gave me great support and confidence. Without their encouragement and support, this paper would not have been completed so smoothly.

\section{REFERENCES}

[1] DSM-5: The Ten Personality Disorders: Cluster B. Mental Help DSM5 The Ten Personality Disorders Cluster B Comments. (n.d.). DOI: https://www.mentalhelp.net/personalitydisorders/cluster- 
b\#: :text=Persons \%20with\%20Histrionic\%20Pers onality $\% 20$ Disorder,not $\% 20$ the $\% 20$ center $\% 20$ of $\%$ 20attention.

[2] Glenn, A. L., Johnson, A. k., \& Raine, A. (n.d.). (PDF) antisocial personality disorder: A current review. ResearchGate.

DOI: https://www.researchgate.net/publication/2587029 58

Antisocial_Personality_Disorder_A_Current_Revi ew.

[3] Mayo clinic. (2019, December 10). Antisocial personality disorder. Mayo Clinic. DOI: https://www.mayoclinic.org/diseasesconditions/antisocial-personalitydisorder/diagnosis-treatment/drc-20353934.

[4] Tuvblad, C., Narusyte, J., Grann, M., Sarnecki, J., \& Lichtenstein, P. (2011, March 23). The genetic and environmental etiology of antisocial behavior from childhood to emerging adulthood. Behavior Genetics. DOI: https://link.springer.com/article/10.1007/s10519011-9463-4.

[5] Ferguson, C. J. (n.d.). Genetic contributions to antisocial personality and behavior: A meta-analytic review from an evolutionary perspective. Taylor \& Francis.

DOI: https://www.tandfonline.com/doi/abs/10.1080/002 24540903366503.

[6] Crowe, R. R. An adoption study of antisocial personality. Archives of General Psychiatry, vol. 31(6), $\quad 1974, \quad 785 . \quad$ DOI: https://doi.org/10.1001/archpsyc.1974.0176018002 7003

[7] Van den Bree, M. B. M., Svikis, D. S., \& Pickens, R. W. Genetic influences in antisocial personality and drug use disorders. Drug and Alcohol Dependence, vol. 49(3), 1998, pp.177-187. DOI: https://doi.org/10.1016/s0376-8716(98)00012-x

[8] DeLisi, M., Drury, A. J., \& Elbert, M. J. (2019, April 30). The etiology of antisocial personality disorder: The differential roles of adverse childhood experiences and childhood psychopathology. Comprehensive Psychiatry. DOI: https://www.sciencedirect.com/science/article/pii/S 0010440X19300215.

[9] Paris, J., \& MD. (n.d.). Antisocial personality disorder: A biopsychosocial model. Canadian journal of psychiatry. Revue canadienne de psychiatrie.

DOI:
[10] Moran, P. (n.d.). The epidemiology of antisocial personality disorder. Social Psychiatry and Psychiatric Epidemiology. DOI: https://link.springer.com/article/10.1007\%2Fs0012 70050138.

[11] Zimmerman, M., \& Last full review/revision May 2021| Content last modified May 2021. (2021, May 21). Overview of personality disorders - psychiatric disorders. Merck Manuals Professional Edition. DOI:

https://www.merckmanuals.com/professional/psyc hiatric-disorders/personality-disorders/overviewof-personality-disorders. https://pubmed.ncbi.nlm.nih.gov/8705966/. 